\title{
Breaking RSA Generically is Equivalent to Factoring*
}

\author{
Divesh Aggarwal Ueli Maurer \\ Department of Computer Science \\ ETH Zurich \\ CH-8092 Zurich, Switzerland \\ \{divesha, maurer\}@inf .ethz.ch
}

\begin{abstract}
Let $N$ be a random variable distributed according to some appropriate distribution over the set of products of two primes, such that factoring $N$ is believed to be hard. The RSA assumption states that, given an a chosen uniformly at random from $\mathbb{Z}_{N}$ and an $e \in \mathbb{N} \backslash\{1\}$, it is computationally hard to find an $x \in \mathbb{Z}_{N}$ such that $x^{e}-a \equiv 0$ $(\bmod N)$.

When complexity-theoretic (relative) lower bounds for certain cryptographic problems in a general model of computation seem to elude discovery, a common practice in cryptography is to give proofs of computational security in meaningful restricted models of computation. An example of such a restricted model that is interesting in cryptography is the generic group model that has been used for proving lower bounds for the discrete logarithm problem and other related problems. A generic model captures the cases in which an algorithm does not exploit the bit representation of the elements other than for testing equality.

In this paper, we prove that the problem of factoring $N$ can be efficiently reduced to solving the RSA problem on $\mathbb{Z}_{N}$ in the generic ring model of computation, where an algorithm can perform ring operations, inverse ring operations, and test equality. This provides evidence towards the soundness of the RSA encryption and digital signature scheme, in particular showing that under the factoring assumption, they are not vulnerable to certain kinds of cryptanalytic attacks.
\end{abstract}

Keywords: Generic Algorithms, Reductions, Factoring, RSA

*A preliminary version of this paper appeared in the proceedings of EUROCRYPT 2009 [1]. 


\section{Introduction}

The two most fundamental reduction problems in number-theoretic cryptography are to prove or disprove that breaking the RSA system [18] is as hard as factoring integers and that breaking the Diffie-Hellman protocol [7] is as hard as computing discrete logarithms. While the second problem has been solved to a large extent $[13,16,3]$, not much is known about the first for general models of computation. In this paper, we show that breaking RSA generically is as hard as factoring the RSA modulus.

\subsection{RSA and Factoring}

In the RSA public key encryption scheme [18], the message $x \in \mathbb{Z}_{n}$, where $n$ is a product of two primes, is encrypted as $x^{e}(\bmod n)$, where $e>1$ is an integer. The security of this scheme relies on the assumption that, given $a$, it is hard to find $x$ such that $x^{e}-a \equiv 0$ $(\bmod n)$.

Before we state the RSA assumption formally, we introduce the following notation. Let $\kappa$ denote the security parameter. Let $N_{\kappa}$ be a $\kappa$-bit random variable chosen according to any distribution from the set of products of two distinct primes, and let $e_{\kappa}>1$ be an integer, whose length is bounded from above by a polynomial in $\kappa$. Our main result is a reduction which works for all $n=p q$ and all $e$. This is the reason we do not specify the distribution of $N_{\kappa}$ or the choice of $e_{\kappa} \cdot{ }^{1}$

We state some assumptions below whose relations we study in this paper:

- Factoring Assumption for $\left(N_{\kappa}\right)_{\kappa \in \mathbb{N}}$ : There exists no probabilistic polynomial-time algorithm that, given $N_{\kappa}$, finds a non-trivial factor of $N_{\kappa}$ with non-negligible probability. ${ }^{2}$

- RSA Assumption for $\left(N_{\kappa}, e_{\kappa}\right)_{\kappa \in \mathbb{N}}$ : There exists no probabilistic polynomial-time algorithm that, given the pair $\left(N_{\kappa}, e_{\kappa}\right)$ and an element $a$ chosen uniformly at random from $\mathbb{Z}_{N_{\kappa}}$, computes $x \in \mathbb{Z}_{N_{\kappa}}$ such that $x^{e_{\kappa}}-a \equiv 0\left(\bmod N_{\kappa}\right)$ with non-negligible probability.

The probability of success of the algorithm in the above definitions is taken over $N_{\kappa}$, $a$, and the randomness of the algorithm.

It is easy to see that if the RSA assumption holds then the factoring assumption holds. However it is a long-standing open problem whether the converse is true. Since no progress has been made for general models of computation, it is interesting to investigate reasonable

\footnotetext{
${ }^{1}$ Note that in practice, for RSA, one usually generates $e$, possibly randomly, depending on the choice of the modulus $n$. Even though we consider $e_{\kappa}$ to be independent of the distribution of $N_{\kappa}$, our result will extend to the case where $e_{\kappa}$ is a random variable that depends on $N_{\kappa}$.

${ }^{2} \mathrm{~A}$ function $f(\kappa)$ is considered a non-negligible function in $\kappa$ if there exists $c>0$ and $k_{0} \in \mathbb{N}$ such that for all $\kappa>k_{0},|f(\kappa)|>\frac{1}{\kappa^{c}}$. The terms polynomial-time and negligible in the definitions of these assumptions are with respect to the security parameter $\kappa$.
} 
restricted models of computation and prove that in such a model factoring is equivalent to the RSA problem. In a restricted model one assumes that only certain kinds of operations are allowed. Shoup [19], based on the work of Nechaev [17], introduced the concept of generic algorithms which are algorithms that do not exploit any property of the representation of the elements. They proved lower bounds on the complexity of computing discrete logarithms in cyclic groups in the context of generic algorithms. Maurer [14] provided a simpler and more general model for analyzing representation-independent algorithms. In this work, we consider the following assumption that breaking RSA is hard in the generic ring model.

- Generic RSA Assumption for $\left(N_{\kappa}, e_{\kappa}\right)_{\kappa \in \mathbb{N}}$ : There exists no probabilistic polynomialtime generic ring algorithm (a class of algorithms that we will formally define later) that, given the pair $\left(N_{\kappa}, e_{\kappa}\right)$ and an element $a$ chosen uniformly at random from $\mathbb{Z}_{N_{\kappa}}$, computes $x \in \mathbb{Z}_{N_{\kappa}}$ such that $x^{e_{\kappa}}-a \equiv 0\left(\bmod N_{\kappa}\right)$ with non-negligible probability.

The following is the main result of this paper.

Theorem 1 For all $\kappa>0$, let $N_{\kappa}$ be a random variable distributed over $\kappa$-bit integers that are products of two primes. If the factoring assumption for $\left(N_{\kappa}\right)_{\kappa \in \mathbb{N}}$ holds, then for all integers $e_{\kappa} \in\left[2,2^{\text {poly }(\kappa)}\right]$, the generic $R S A$ assumption holds for $\left(N_{\kappa}, e_{\kappa}\right)_{\kappa \in \mathbb{N}}$.

\subsection{The Generic Model of Computation}

We give a brief description of the model of [14]. The model is characterized by a black-box $\mathbf{B}$ which can store values from a certain set $\mathcal{Z}$ in internal state variables $V_{0}, V_{1}, V_{2}, \cdots$. The initial state (the input of the problem to be solved) consists of the values of $\left[V_{0}, \ldots, V_{\ell}\right]$ for some positive integer $\ell$, which are set according to some probability distribution (e.g., the uniform distribution).

The black box $\mathbf{B}$ allows two types of operations:

- Computation operations. For a set $\Pi$ of operations of some arities on $\mathcal{Z}$, a computation operation consists of selecting $f \in \Pi$ (say $t$-ary) as well as the indices $i_{1}, \ldots, i_{t+1}$ of $t+1$ state variables. $\mathbf{B}$ computes $f\left(V_{i_{1}}, \ldots, V_{i_{t}}\right)$ and stores the result in $V_{i_{t+1}}$.

- Relation Queries. For a set $\Sigma$ of relations (of some arities) on $\mathcal{Z}$, a query consists of selecting a relation $\rho \in \Sigma$ (say $t$-ary) as well as the indices $i_{1}, \ldots, i_{t}$ of $t$ state variables. The query is replied by the binary output $\rho\left(V_{i_{1}}, \ldots, V_{i_{t}}\right)$ that takes the value 1 if the relation is satisfied, and 0 otherwise.

For this paper, we only consider the case $t=2$ and the only relation queries we consider are equality queries.

An algorithm in this model is characterized by its interactions with the black box $\mathbf{B}$. The algorithm inputs operations (computation operations and relation queries) to the black 
box, and the replies to the relation queries are input to the algorithm. The complexity of an algorithm for solving any problem can be measured by the number of operations it performs on $\mathbf{B}$.

For this paper, the set $\mathcal{Z}$ is $\mathbb{Z}_{N}$. Moreover, $\ell=1$, and $V_{0}$ is always set to be the unit element 1 of $\mathbb{Z}_{N}$, and $V_{1}$ is the value $a$. A generic ring algorithm (GRA) is an algorithm that is just allowed to perform the ring operations, i.e., addition and multiplication as well as the inverse ring operations (subtraction and division), and to test for equality (i.e., make an equality query). In this model, for example, GRAs on $\mathbb{Z}_{N}$ correspond to $\Pi=\{+,-, \cdot, /\}$ and $\Sigma=\{e q\}$, where $e q$ stands for equality queries. A straight-line program $(S L P)$ on $\mathbb{Z}_{N}$, which is a deterministic algorithm that is just allowed to perform ring operations, corresponds to the case where $\Sigma$ is the empty set, i.e., no equality tests are possible.

Some results like [11] in the literature are restricted in that they exclude the inverse operations, but since these operations are easy ${ }^{3}$ to perform in $\mathbb{Z}_{N}$, they should be included as otherwise the results are of relatively limited interest.

\subsection{Discussion and Relevance of the Generic Model of Computation}

Since Shoup's result [19] on proving lower bounds for computing discrete logarithms and some related problems in the generic group model (where the only allowed operations are the group operations and equality queries), it has been a well accepted approach to give proofs of hardness of problems relevant in cryptography in the generic group/ring model.

Three kinds of problems can be considered in the generic group/ring model: extraction problems, computation problems and decision problems.

Extraction problems are problems where the task of the algorithm is to extract the input $x$ from the black-box. An example of this is the discrete logarithm problem, as has been explained in [14]. Other examples of the extraction problem in related models have been considered in $[3,15,2]$.

Decision problems are problems of computing some predicate (function) of the input which evaluates to either 0 or 1 . In this case, a generic algorithm tries to guess the bit based on its interactions with the blackbox. An example of this in the generic group model is the Decisional Diffie Hellman problem.

The only possible way to guess the output of a decision problem is based on the result of the relation queries. In the generic ring model described in Section 1.2, the only relation queries allowed are equality queries. Thus, for the ring $\mathbb{Z}_{N}$, as we show in Lemma 5 , if the input is chosen uniformly at random from $\mathbb{Z}_{N}$, then if we can obtain any non-trivial information from an equality query with non-negligible probability, then we can use this to factor $N$. Therefore, under the assumption that factoring $N$ is hard, there does not exist any GRA for solving any decision problem on the ring $\mathbb{Z}_{N}$ for an input chosen uniformly

\footnotetext{
${ }^{3}$ Division of an element $a$ by $b$ for $a, b \in \mathbb{Z}_{N}$ can be performed easily by first computing $b^{-1}$ using Euclid's algorithm and then computing $a \cdot b^{-1}$ in $\mathbb{Z}_{N}$.
} 
at random from $\mathbb{Z}_{N}$. Thus, even problems as simple as computing the least significant bit of a random input in $\mathbb{Z}_{N}$ is hard with respect to generic ring algorithms. In particular, computing the Jacobi symbol is an example of a problem that is easy to solve in general, but is hard in the generic ring model as has also been pointed out in [9].

This may at first seem to suggest that a result showing the hardness of a certain problem with respect to generic ring algorithms is of no significance. However, computation problems still remain an interesting class of problems in the generic ring model of computation. The computation problems are problems where the algorithm is required to compute a function of the input that results in a set of elements of the underlying group/ring structure. The algorithm is successful if it is able to compute this set of elements inside the black-box using the allowed operations and relation queries. An example of a computation problem in the generic group model is the Computational Diffie Hellman problem.

There exist (maybe inefficient) generic algorithms for solving almost all computation problems. In particular, consider the RSA problem in the ring of integers modulo $N=p q$. There is a trivial GRA that solves the RSA problem by computing $x^{e}$ for different values of $x$ from $2,3, \ldots$ until an $x$ is obtained such that $x^{e}=a$. Thus it is interesting to obtain a result that shows the impossibility of getting an efficient GRA under a plausible assumption like the assumption that factoring is hard.

A result in the generic model makes more sense than, for example, results in the monotone circuit model in complexity theory where one is allowed only AND and OR but no NOT gates, since there are instances where generic algorithms are the best known algorithms e.g., discrete logarithms problem over elliptic curves, while such instances do not exist for the monotone circuit model. In particular, a result in this model rules out certain classes of cryptanalytic attacks for breaking the corresponding cryptosystem.

Motivated by the fact that decision problems like the Jacobi symbol are hard in the generic model and easy in general, one might want to consider a more general model than the generic ring model of computation where one is, for example, given oracle access to the Jacobi symbol. This can be modeled nicely by allowing another relation query corresponding to whether the Jacobi symbol is 0 or 1 .

\subsection{Comparison with Related Work}

Research on the relation between RSA and factoring comes in two flavours. There have been results giving evidence against (e.g. [4, 10]) and in favour of (e.g. [5, 11]) the assumption that breaking RSA is equivalent to factoring.

Boneh and Venkatesan [4] showed that any SLP that factors $N$ by making at most a logarithmic number of queries to an oracle solving the Low-Exponent RSA (LE-RSA) problem (the RSA problem when the public exponent $e$ is small) can be converted into a real polynomial-time algorithm for factoring $N$. This means that if factoring is hard, then there exists no straight-line reduction from factoring to LE-RSA that makes a small number of queries to the LE-RSA oracle. Joux et al [10] showed that given access to an oracle that 
computes $e$-th roots of numbers of the form $x+c$ for a fixed $c$ and any $x$, computing arbitrary $e$-th roots modulo $N$ is easier than factoring $N$.

Brown [5] showed that if factoring is hard then the LE-RSA problem is intractable for SLPs with $\Pi=\{+,-, \cdot\}$. More precisely, he proved that an efficient SLP for breaking LE-RSA can be transformed into an efficient factoring algorithm. Leander and Rupp [11] generalized the result of [5] to GRAs which, as explained above, can test the equality of elements. Again, division is excluded $(\Pi=\{+,-, \cdot\})$.

Another theoretical result about the hardness of the RSA problem is due to Damgård and Koprowski [6]. They studied the problem of root extraction in finite groups of unknown order and proved that the RSA problem is intractable with respect to generic group algorithms. This corresponds to excluding addition, subtraction and division from the set of operations $(\Pi=\{\cdot\})$.

Our results generalize the previous results in several ways. In fact, Theorem 1 appears to be the most general statement about the equivalence of breaking RSA in a generic ring model and factoring.

- First, compared to $[5,11]$, we consider the full-fledged RSA problem (not only LE-RSA) with exponent $e$ of arbitrary size, even with bit-size much larger than that of $N$.

- Second, compared to $[6,5,11]$, we consider the unrestricted set of ring operations, including division. This generalization is important since there are problems that are easy to solve in our generic ring model but are provably hard to solve using the model without division ${ }^{4}$. Actually, as has been pointed out in [5], computing the multiplicative inverse of a random element in $\mathbb{Z}_{N}$ generically is hard if $\Pi=\{+,-, \cdot\}$.

The problem we solve has been stated as an open problem in [6] and [5].

\section{Preliminaries}

\subsection{Straight-Line Programs and Generic Ring Algorithms}

In this section we define GRAs and SLPs (as a special case) as (syntactic) mathematical objects. Then we define the semantics of the GRA (SLP) in terms of what is computed at each step, and finally we define the partial function $\mathbb{Z}_{n} \rightarrow \mathbb{Z}_{n}$ computed by the GRA (SLP) when it is run for the ring $\mathbb{Z}_{n}$.

An SLP (for rings) is a deterministic algorithm that performs a sequence of ring operations. Thus an SLP corresponds to $\Pi=\{+,-, \cdot, /\}$ and $\Sigma=\{\}$ in the model of [14]. More precisely, an SLP is a sequence of operations, where the $k$-th operation is of the form $(i, j, \circ)$ for $0 \leq i, j<k$ and $\circ \in\{+,-, \cdot, /\}$. The result of the $k$-th step is defined as the result

\footnotetext{
${ }^{4}$ In [5], the author has mentioned and given justification for the fact that most results of his paper will extend to SLPs with division.
} 
of applying $\circ$ to the $i$-th and the $j$-th intermediate results. To be compatible with the definition of a GRA, we define an SLP as a labeled path (a graph) rather than a sequence:

Definition 1 An L-step SLP $S$ is a partially labeled path $v_{0}, \ldots, v_{L}$ where $v_{0}, v_{1}$ are unlabeled and $v_{k}$ for $k \in[2, L]$ carries a label of the form $\left(v_{i}, v_{j}, \circ\right)$ for $0 \leq i, j<k$ and $\circ \in$ $\{+,-, \cdot, /\}$.

A deterministic generic ring algorithm is a generalized SLP that also allows equality queries. Hence a GRA corresponds to a labeled tree, where branches in the tree correspond to equality queries, where the left [right] child of a branching vertex corresponds to the equality test being [not] satisfied. An equality query must specify which two intermediate results (i.e., which vertices in the graph) are to be compared. Formally:

Definition 2 An $L$-step deterministic $G R A G$ is a depth- $L$ partially vertex-labeled and edge-labeled binary tree where the root vertex and its child are unlabeled and each has one outgoing edge, and the remaining tree has two kind of vertices: branching vertices and non-branching vertices. A branching vertex $v$ has two outgoing edges labeled 0 (for left edge) and 1 (for right edge), and $v$ carries a label of the form $(u, w)$, where $u, w$ are some non-branching vertices in the path from the root to $v$. A non-branching vertex $v$ has one outgoing edge and $v$ has a label of the form $(u, w, \circ)$, where $u, w$ are some non-branching vertices in the path from the root to $v$, and $\circ \in\{+,-, \cdot, /\}$.

Note that an SLP can be seen as a special case of a GRA with no branching vertices. Next, we define a randomized GRA as a further generalization of a deterministic GRA, where the choice of the operation at each step is randomized.

Definition 3 A randomized GRA is a random variable whose values are deterministic GRAs.

A GRA (or SLP) without division operation can naturally be interpreted as computing a polynomial in $\mathbb{Z}[x]$ at each non-branching vertex, where by definition the root and the child of the root are labeled by polynimials 1 and $x$, respectively. (Here $x$ stands for the indeterminate of the polynomial. For a concrete argument $a$ in the ring, $x$ will be thought of being set to a.) Note that these polynomials are defined over $\mathbb{Z}[x]$, no matter to which particular ring $R$ (e.g. $R=\mathbb{Z}_{n}$ ) it is thought of as being applied. If the GRA contains division operations, then each non-branching vertex corresponds naturally to a pair of polynomials in $\mathbb{Z}[x]$, the numerator and the denominator polynomial. Note that we do not yet interpret this pair as a (rational) function and hence the possible problem of a division by 0 does not arise at this point.

Definition 4 For a GRA $G$ (or SLP $S$ ) and non-branching vertex $v$, the pair $\left(P_{v}^{G}(x), Q_{v}^{G}(x)\right.$ ) of polynomials in $\mathbb{Z}[x]$ associated with $v$ is defined inductively, as follows:

1. The root has associated the pair $(1,1)$, and the child of the root the pair $(x, 1)$. 
2. For each non-branching vertex $v$, labeled with operation $(u, w, o)$, we have

$$
\left(P_{v}^{G}(x), Q_{v}^{G}(x)\right)= \begin{cases}\left(P_{u}^{G}(x) \cdot Q_{w}^{G}(x)+P_{w}^{G}(x) \cdot Q_{u}^{G}(x), Q_{u}^{G}(x) \cdot Q_{w}^{G}(x)\right) & \text { if } \circ \text { is }+ \\ \left.P_{u}^{G}(x) \cdot Q_{w}^{G}(x)-P_{w}^{G}(x) \cdot Q_{u}^{G}(x), Q_{u}^{G}(x) \cdot Q_{w}^{G}(x)\right) & \text { if } \circ \text { is - } \\ \left.P_{u}^{G}(x) \cdot P_{w}^{G}(x), Q_{u}^{G}(x) \cdot Q_{w}^{G}(x)\right) & \text { if } \circ \text { is } ~ \\ \left.P_{u}^{G}(x) \cdot Q_{w}^{G}(x), Q_{u}^{G}(x) \cdot P_{w}^{G}(x)\right) & \text { if } \circ \text { is } /\end{cases}
$$

If the GRA is an SLP $S$, then the final pair of polynomials (i.e., one that corresponding to the last vertex) is unique and we call it $\left(P^{S}, Q^{S}\right)$.

It follows from the definitions that any $L$-step SLP $S$ of length $L$ can be "converted" into an SLP $S^{\prime}$ of length at most $4 L$ that computes both $\left(P^{S}, 1\right)$ and $\left(Q^{S}, 1\right)$. A result similar to this but with a factor of 6 instead of 4 has been proven independently in [8].

Lemma 1 For any $L$-step $S L P S$, there exists a $4 L$-step $S L P S^{\prime}$ and some indices $r, t \leq 4 L$ such that $\left(P_{v_{r}}^{S^{\prime}}, Q_{v_{r}}^{S^{\prime}}\right)=\left(P_{L}^{S}, 1\right)$ and $\left(P_{v_{t}}^{S^{\prime}}, Q_{v_{t}}^{S^{\prime}}\right)=\left(Q_{L}^{S}, 1\right)$.

For a fixed (partially invertible) ring $R$, one can associate to every vertex $v$ of a GRA $G$ the partial function $f_{v}^{G}$ computed at that vertex, as explained in the definition below. Moreover, executing a GRA for a given ring $R$ and a given argument $a \in R$ means to compute along a path, starting at the root, where each equality test (comparing the values computed at two previous vertices) determines which branch is taken in the corresponding branching vertex. Such a computation ends in a leaf $\ell$ of the tree, where the leaf $\ell$ that is reached depends on the argument $a$. For argument $a$, the value computed at the reached leaf is defined as the function value $f^{G}(a)$ computed by the GRA. More formally:

Definition 5 For each non-branching vertex $v$ in a GRA $G$ with corresponding pair of polynomials $\left(P_{v}^{G}(x), Q_{v}^{G}(x)\right)$, we associate the function

$$
f_{v}^{G}: R \rightarrow R \cup\{\perp\}: a \mapsto \frac{P_{v}^{G}(a)}{Q_{v}^{G}(a)},
$$

where the function is undefined if $Q_{v}^{G}(a)=0$, which is denoted as $f_{v}^{G}(a)=\perp$, and where $P_{v}^{G}(a)$ and $Q_{v}^{G}(a)$ are evaluated over $R$. Moreover, for an argument $a \in R$, the computation path $v_{0}, v_{1}, \ldots, v_{\ell}$ from the root $v_{0}(a)$ to a leaf $v_{\ell}(a)=: \Lambda(a)$ is defined by taking, for each equality test of the form $(u, w)$, the edge labeled 0 if $f_{u}^{G}(a)=f_{w}^{G}(a)$, and the edge labeled 1 if $f_{u}^{G}(a) \neq f_{w}^{G}(a)$. The partial function $f^{G}$ computed by $G$ is defined as

$$
f^{G}: R \rightarrow R \cup\{\perp\}: a \mapsto f_{\Lambda(a)}^{G} .
$$

We only consider rings of the form $R=\mathbb{Z}_{n}$, and for this case the function computed by GRA $G$ will be denoted as $f^{G, n}: \mathbb{Z}_{n} \rightarrow \mathbb{Z}_{n}$. 


\subsection{Mathematical Preliminaries}

In this section we introduce some notations and prove some results about the mathematical structures used in this paper.

For any event $\mathrm{E}$, we denote the probability of $\mathrm{E}$ by $\operatorname{Pr}(\mathrm{E})$. For any integer $n, \mathbb{Z}_{n}[x]$ denotes the ring of polynomials in $x$ with coefficients in $\mathbb{Z}_{n}$. For $h(x) \in \mathbb{Z}_{n}[x], \mathbb{Z}_{n}[x] /(h(x))$ denotes the quotient ring $\mathbb{Z}_{n}[x]$ by a principal ideal generated by $h(x)$.

Definition 6 For any integer $n$ and any partial function $f: \mathbb{Z}_{n} \mapsto \mathbb{Z}_{n} \cup\{\perp\}$, we define the following.

- Let $\eta_{n}(f)$ denote the fraction of elements $a$ in $\mathbb{Z}_{n}$ such that $f(a)$ has a non-trivial greatest common divisor with $n$ :

$$
\eta_{n}(f):=\frac{\left|\left\{a \in \mathbb{Z}_{n} \mid f(a) \neq \perp \wedge \operatorname{gcd}(f(a), n) \notin\{1, n\}\right\}\right|}{n} .
$$

- Let $\nu_{n}(f)$ denote the fraction of roots of $f$ in $\mathbb{Z}_{n}$, i.e.,

$$
\nu_{n}(f):=\frac{\left|\left\{a \in \mathbb{Z}_{n} \mid f(a)=0\right\}\right|}{n} .
$$

- For any deterministic GRA $G$, and any function $g: \mathbb{Z}_{n} \mapsto \mathbb{Z}_{n}$, let $\lambda_{n}(G, g)$ be the success probability of $G$ in computing $g$, defined as:

$$
\lambda_{n}(G, g):=\nu_{n}\left(f^{G, n}(x)-g(x)\right),
$$

where $f^{G, n}(x)-g(x)=\perp$ if $f^{G, n}(x)=\perp$.

For $n \in \mathbb{N}$ and $e>1$, such that $\operatorname{gcd}(e, \phi(n))=1$, we denote by $x^{1 / e}: \mathbb{Z}_{n} \mapsto \mathbb{Z}_{n}$, the function that maps $a \in \mathbb{Z}_{n}$ to $b \in \mathbb{Z}_{n}$ such that $b^{e}=a$.

We prove a few results that we will need later. These are similar to what was used in $[5,11]$.

Lemma 2 Let $n=p q$ be a product of two primes. For any $P(x) \in \mathbb{Z}_{n}[x]$ and for any $\delta>0$, if $\nu_{n}(P) \in[\delta, 1-\delta]$, then $\eta_{n}(P) \geq \delta^{\frac{3}{2}}$.

Proof. We denote $\nu_{p}(P)$ and $\nu_{q}(P)$ by $\nu_{p}$ and $\nu_{q}$, respectively. By the Chinese remainder theorem, $\nu_{n}(P)=\nu_{p} \cdot \nu_{q}$ and $\eta_{n}(P)=\nu_{p}\left(1-\nu_{q}\right)+\nu_{q}\left(1-\nu_{p}\right)$. Using $\delta \leq \nu_{p} \cdot \nu_{q} \leq 1-\delta$, we obtain

$$
\begin{aligned}
\eta_{n}(P) & =\nu_{p}+\nu_{q}-2 \nu_{p} \cdot \nu_{q} \\
& \geq 2 \sqrt{\nu_{p} \cdot \nu_{q}}-2 \nu_{p} \cdot \nu_{q} \\
& =2 \sqrt{\nu_{p} \cdot \nu_{q}}\left(1-\sqrt{\nu_{p} \cdot \nu_{q}}\right) \\
& \geq 2 \sqrt{\delta}(1-\sqrt{1-\delta}) \\
& \geq 2 \sqrt{\delta}\left(1-\left(1-\frac{\delta}{2}\right)\right) \\
& =\delta^{\frac{3}{2}}
\end{aligned}
$$


Lemma 3 Let $p$ be a prime and $d$ be a positive integer. A random monic polynomial $f(x) \in$ $\mathbb{Z}_{p}[x]$ of degree $d$ is irreducible in $\mathbb{Z}_{p}[x]$ with probability at least $\frac{1}{2 d}$ and has a root in $\mathbb{Z}_{p}$ with probability at least $1 / 2$.

Proof. From the distribution theorem of monic polynomials (see, e.g., [12]) it follows that the number of monic irreducible polynomials of degree $d$ over $F_{p}$ is at least $\frac{p^{d}}{2 d}$. Therefore $f(x)$ is an irreducible polynomial over $\mathbb{Z}_{p}$ with probability at least $\frac{1}{2 d}$.

The number of monic polynomials over $\mathbb{Z}_{p}$ with at least one root is:

$$
\sum_{l=1}^{d}(-1)^{l-1}\left(\begin{array}{l}
p \\
l
\end{array}\right) p^{d-l}
$$

This can be seen by applying the principle of inclusion and exclusion. The terms in this summation are in decreasing order of their absolute value. So, taking the first two terms, this sum is greater than $\left(\begin{array}{l}p \\ 1\end{array}\right) p^{d-1}-\left(\begin{array}{l}p \\ 2\end{array}\right) p^{d-2}$ which is greater than $\frac{p^{d}}{2}$. Hence the probability that $f(x)$ has a root in $\mathbb{Z}_{p}$ is at least $1 / 2 .^{5}$

\section{The Main Reduction}

In this section, we prove the following theorem.

Theorem 2 For all $\mu \in(0,1)$, there exists a probabilistic algorithm $\mathcal{A}$ that takes as input an integer $n=p q$ that is a product of two primes, an integer $e>1$, and an L-step randomized $G R A \mathcal{G}$, runs in time polynomial in $\log n, \log e, L$, and $\frac{1}{\mu}$, such that whenever

$$
\mathrm{E}_{\mathcal{G}}\left[\lambda_{n}\left(\mathcal{G}, x^{1 / e}\right)\right] \geq \mu
$$

then $\mathcal{A}$ computes a factor of $n$ with probability at least $1 / 2$.

It is easy to see that Theorem 1 is an immediate corollary of Theorem 2.

Throughout this section, we write $f(x) \equiv 0(\bmod n)$ if $f$ is the constant 0 -function modulo $n$, and for a (possibly partial) function $f$ we write $f(x) \not \equiv 0(\bmod n)$ otherwise.

In Section 3.1, we show that an SLP that computes $e$-th roots modulo $n$ with nonnegligible probability can be used to factor $n$. Then, in Section 3.2, we show that from a deterministic GRA that computes $e$-th roots, we can either obtain an SLP that computes $e$ th roots, or directly obtain a factor of $n$. In Section 3.3, we combine the results of Section 3.1 and 3.2 to show that a randomized GRA that computes $e$-th roots can be used to factor $n$.

\footnotetext{
${ }^{5}$ Note that, by a careful analysis, it is possible to prove a better lower bound on the probability that $f(x)$ has a root in $\mathbb{Z}_{p}$ but a lower bound of $1 / 2$ is sufficient for our purpose.
} 


\subsection{The Proof for Straight-Line Programs}

We first give an algorithm that factors $n$ given access to an SLP that computes a non-trivial polynomial that is 0 modulo $n$.

For $b(x), c(x) \in \mathbb{Z}_{n}[x]$, let $\operatorname{gcd}_{p}(b(x), c(x))$ and $\operatorname{gcd}_{q}(b(x), c(x))$ be the greatest common divisor of the polynomials modulo $p$ and $q$, respectively. The following proposition is easy to see.

Proposition 1 Let $b(x), c(x) \in \mathbb{Z}_{n}[x]$. If $\operatorname{deg}\left(\operatorname{gcd}_{p}(b(x), c(x))\right) \neq \operatorname{deg}\left(\operatorname{gcd}_{q}(b(x), c(x))\right)$, then Euclid's algorithm on $\mathbb{Z}_{n}[x]^{6}$ with input $b(x)$ and $c(x)$ yields a non-trivial non-invertible element of $\mathbb{Z}_{n}$.

We denote by $H(b(x), c(x))$ the non-trivial non-invertible element output when Euclid's algorithm is executed on $Z_{n}[x]$ with input $b(x)$ and $c(x)$.

Lemma 4 There exists a randomized algorithm (Algorithm 1) that takes as input $n=p q$, where $p, q>3$ are primes, $L \in \mathbb{N}$, and an $L$-step $S L P S$, runs in time $O\left(L^{3} \log ^{2} n\right)$, and does the following. If $\left(P^{S}(x), Q^{S}(x)\right)=(f(x), 1)$, and $f(x) \not \equiv 0 \bmod n$, then Algorithm 1 returns a factor of $n$ with probability at least $\frac{\nu_{n}(f)}{8 L}$.

Proof. Consider Algorithm 1. By Proposition 1, if Euclid's algorithm fails in step 5 or step 6 , then we get a factor of $n$.

Now we compute the success probability of the algorithm. Without loss of generality, we assume that $f(x) \not \equiv 0 \bmod q$. By Lemma 3, the probability that $h(x)$ is irreducible modulo $q$ and has a root modulo $p$ is at least $\frac{1}{2 L} \cdot \frac{1}{2}=\frac{1}{4 L}$. We assume that this is the case for the rest of the proof and all subsequent probabilities are computed conditioned on this event.

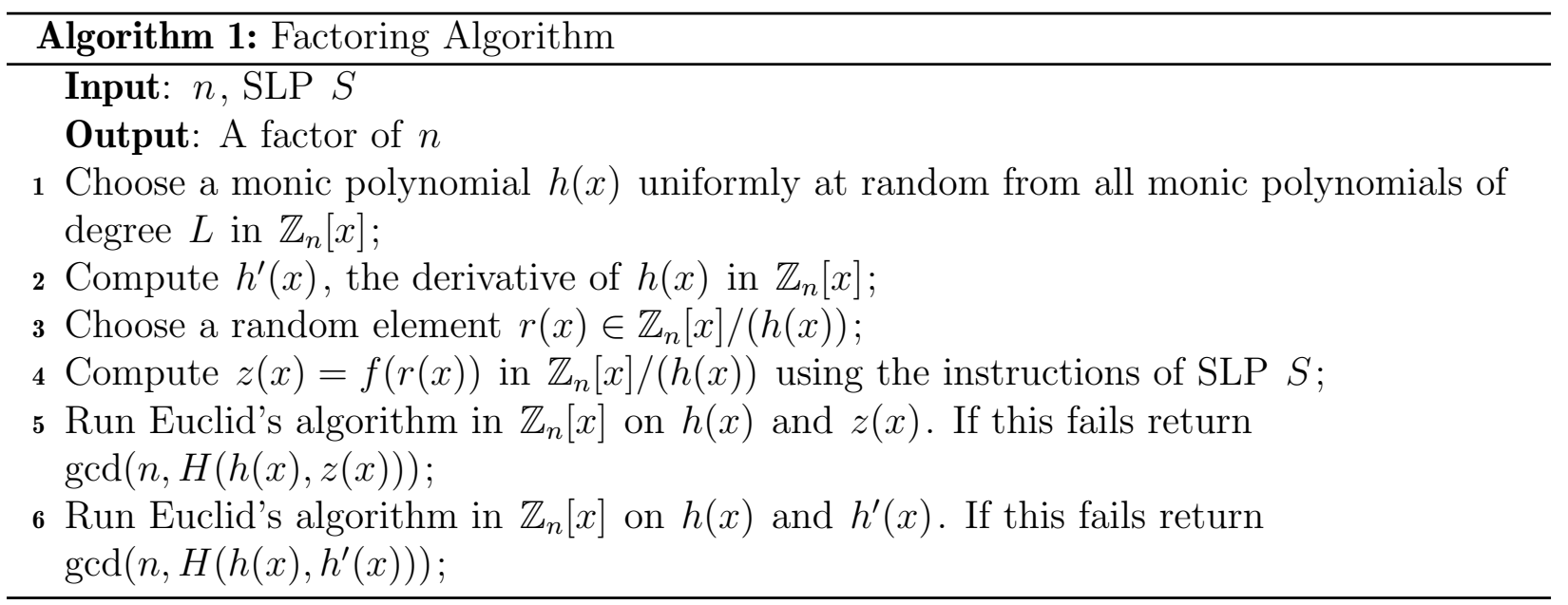

Let this root of $h(x)$ modulo $p$ be $s$. Therefore $(x-s)$ divides $h(x)$ in $\mathbb{Z}_{p}[x]$. We analyze two cases.

\footnotetext{
${ }^{6}$ Note that $\mathbb{Z}_{n}[x]$ is not a Euclidean domain
} 
- CASE 1: $(x-s)^{2}$ divides $h(x)$ in $\mathbb{Z}_{p}[x]$.

This implies that $(x-s)$ divides $\operatorname{gcd}_{p}\left(h(x), h^{\prime}(x)\right)$. However, since $h(x)$ is irreducible in $\mathbb{Z}_{q}[x]$, the degree of $\operatorname{gcd}_{q}\left(h(x), h^{\prime}(x)\right)$ is 0 . Therefore $\operatorname{gcd}_{p}\left(h(x), h^{\prime}(x)\right)$ and $\operatorname{gcd}_{q}\left(h(x), h^{\prime}(x)\right)$ have different degree, which implies, by Proposition 1, that Euclid's algorithm on $h(x)$ and $h^{\prime}(x)$ fails and hence step 6 yields a factor of $n$.

- CASE 2: $(x-s)^{2}$ does not divide $h(x)$ in $\mathbb{Z}_{p}[x]$.

Let $h(x)=h_{1}(x) \cdot(x-s)$ in $\mathbb{Z}_{p}[x]$. Then:

$\mathbb{Z}_{n}[x] /(h(x)) \cong \mathbb{Z}_{p}[x] /(h(x)) \times \mathbb{Z}_{q}[x] /(h(x)) \cong \mathbb{Z}_{p}[x] /(x-s) \times \mathbb{Z}_{p}[x] /\left(h_{1}(x)\right) \times \mathbb{F}_{q^{L}}$,

because $\mathbb{Z}_{q}[x] /(h(x)) \cong \mathbb{F}_{q^{L}}$ (the finite field containing $q^{L}$ elements) as $h(x)$ is irreducible in $\mathbb{Z}_{q}[x]$ by our assumption.

We identify the elements in the quotient ring by the polynomial representing the corresponding element in the quotient ring.

Under this isomorphism, let $r(x)$ and $z(x)$ map to the triple

$$
\left(r(s) \bmod p, u(x), r_{q}(x)\right) \quad \text { and } \quad\left(z(s) \bmod p, v(x), z_{q}(x)\right),
$$

respectively, where $r_{q}(x)$ and $z_{q}(x)$ are the reductions of $r(x)$ and $z(x)$ modulo $q$. Since $r(x)$ is uniformly random in $\mathbb{Z}_{n}[x] /(h(x)), r(s)$ is uniformly random in $\mathbb{Z}_{p}[x] /(x-$ $s) \cong \mathbb{Z}_{p}$. This implies

$$
\begin{aligned}
\operatorname{Pr}(z(s) \equiv 0 \quad(\bmod p)) & =\operatorname{Pr}(f(r(s)) \equiv 0 \quad(\bmod p)) \\
& \geq \operatorname{Pr}(f(r(s)) \equiv 0 \quad(\bmod n))=\nu_{n}(f) .
\end{aligned}
$$

Therefore, with probability at least $\nu_{n}(f),(x-s)$ divides $z(x)$ in $\mathbb{Z}_{p}[x]$, which implies $\operatorname{Pr}\left((x-s)\right.$ divides $\left.\operatorname{gcd}_{p}(z(x), h(x))\right) \geq \mu$. Since $r(x)$ is uniformly random in $\mathbb{Z}_{n}[x] /(h(x)), r_{q}(x)$ is uniformly random in $\mathbb{Z}_{q}[x] /(h(x)) \cong \mathbb{F}_{q^{L}}$. A non-zero polynomial over a finite field can have at most as many roots as the degree of the polynomial. Therefore, for random $x$,

$$
\operatorname{Pr}\left(z_{q}(x)=0\right)=\operatorname{Pr}\left(f\left(r_{q}(x)\right)=0\right) \leq \frac{\operatorname{deg}(f)}{q^{L}} \leq \frac{2^{L}}{q^{L}} \leq \frac{1}{2}
$$

for $q>3$. We use the fact that $\operatorname{deg}(f) \leq 2^{L}$. This is because, at each step of the SLP which is either an addition, subtraction, or a multiplication operation of two previously computed polynomials, the degree is bounded by the sum of the degrees of the two polynomials. Thus, by induction, the degree is at most $2^{L}$ after $L$ steps of the SLP. Hence, $\operatorname{Pr}\left(z_{q}(x) \not \equiv 0\right) \geq \frac{1}{2}$. The condition $z_{q}(x) \not \equiv 0$ implies that $\operatorname{gcd}_{q}(z(x), h(x))$ has degree 0 because $h(x)$ is irreducible modulo $q$. 
Therefore the probability that Euclid's algorithm run on $h(x)$ and $z(x)$ fails is at least $\frac{1}{4 L} \cdot \nu_{n}(f) \cdot \frac{1}{2}=\frac{\nu_{n}(f)}{8 L}$.

Now we compute the time complexity of one run of the loop. Generating random $h(x)$ and $r(x)$ and computing the derivative requires $O\left(L \log ^{2} n\right)$ operations in $\mathbb{Z}_{n}$. Each operation in $\mathbb{Z}_{n}[x] /(h(x))$ can be implemented by at most $L^{2} \log ^{2} n$ operations in $\mathbb{Z}_{n}$. The function $f(r(x))=z(x)$ can be computed in time $O\left(L^{2} \log ^{2} n \cdot L\right)=O\left(L^{3} \log ^{2} n\right)$. Euclid's algorithm on $z(x)$ and $h(x)$ and on $h(x)$ and $h^{\prime}(x)$ can be performed by $O\left(L^{2} \log ^{2} n\right)$ operations. Thus, the running time of the algorithm is $O\left(L^{3} \log ^{2} n\right)$.

Corollary 1 There exists an algorithm that takes as input $n=p q$, where $p, q>3$ are primes, an integer $e>1, L \in \mathbb{N}$, and an $L$-step $S L P S$, runs in time $O\left(\left(L^{3}+\log ^{3} e\right) \log ^{2} n\right)$, and returns a factor of $n$ with probability at least $\frac{\lambda_{n}\left(S, x^{1 / e}\right)}{32(L+\log e)}$.

Proof. Consider the polynomial $f(x)=\left(P^{S}(x)\right)^{e}-x \cdot\left(Q^{S}(x)\right)^{e}$. We claim that $f(x) \not \equiv 0$ $(\bmod n)$ if and only if $Q^{S}(x)$ (and hence also $\left.P^{S}(x) \not \equiv 0(\bmod n)\right)$. To see this, assume, to the contrary, that $Q^{S}(x)$ is non-zero but $f(x)$ is zero modulo $n$. This implies $P^{S}(x)$ is also non-zero modulo $n$. Let the leading terms of $P^{S}(x)$ and $Q^{S}(x)$ be $a_{0} x^{d_{0}}$ and $a_{1} x^{d_{1}}$, respectively, where $a_{0}, a_{1}$ are not zero modulo $n$. Note that $d_{0} \cdot e \neq d_{1} \cdot e+1$ since $e>1$. Thus, the leading term of $f(x)$ is either $a_{0} x^{d_{0} \cdot e}$, or $a_{1} x^{d_{1} \cdot e+1}$, and hence is non-zero.

Also, note that for any $a \in \mathbb{Z}_{n},\left(f^{S, n}(a)\right)^{e}-a=0$ if and only if $f(a)=0$ and $Q^{S}(a) \neq$ 0 . Thus, we assume that $Q^{S}(x) \not \equiv 0(\bmod n)$, since otherwise $\lambda_{n}\left(S, x^{1 / e}\right)=0$, and the result is vacuously true. This implies that $f(x)$ is not the zero polynomial modulo $n$, and $\lambda_{n}\left(S, x^{1 / e}\right)=\nu_{n}\left(\left(f^{S, n}(x)\right)^{e}-x\right) \leq \nu_{n}(f)$. Thus it suffices to obtain an algorithm that returns a factor of $n$ with probability $\frac{\nu_{n}(f)}{32(L+\log e)}$.

From Lemma 1 we get that from any $L$-step SLP $S$, we can get a $4 L$-step SLP using only operations $\{+,-, \cdot\}$ that computes $\left(P^{S}, 1\right)$ and $\left(Q^{S}, 1\right)$. Hence we can obtain a $(4 L+4 \log e)$ step SLP that computes the pair $\left(\left(P^{S}(x)\right)^{e}-\left(Q^{S}(x)\right)^{e} \cdot x, 1\right)$. Thus, using Lemma 4 , we get the result.

\subsection{From Deterministic GRAs to SLPs}

In this section we give an algorithm that, given access to a deterministic GRA that computes $e$-th roots, outputs either a factor of $n$ or an SLP that computes $e$-th roots.

Lemma 5 For all $\epsilon>0$ and any function $g: \mathbb{Z}_{n} \mapsto \mathbb{Z}_{n}$, there exists a randomized algorithm (Algorithm 2) that, given $n, L \in \mathbb{N}$, and an L-step deterministic GRA $G$, runs in time $O\left(\frac{L^{7 / 2} \log ^{2} n}{\epsilon^{5 / 2}}\right)$ and, with probability $1-\epsilon$, either outputs a factor of $n$ or an L-step SLP $S$ such that $\lambda_{n}(S, g) \geq \lambda_{n, e}(G, g)-\frac{\epsilon}{2}$. 
Proof. Let $\delta=\frac{\epsilon}{2 L}$. We classify the branching vertices in $G$ into two kinds of vertices extreme and non-extreme vertices. For a branching vertex $v$ labeled with $(u, w)$, if

$$
\nu_{n}\left(P_{u}^{G} \cdot Q_{w}^{G}-P_{w}^{G} \cdot Q_{u}^{G}\right) \in[\delta, 1-\delta]
$$

then we call $v$ a non-extreme vertex and otherwise we call $v$ an extreme vertex.

Let $G_{e x}$ be the tree obtained from $G$ by truncating the sub-tree rooted at $v$ for all non-extreme vertices $v$. Therefore all non-extreme vertices present in $G_{e x}$ are at the leaves. Also, we can assume, without loss of generality, that a leaf vertex of $G$ is not a branching vertex since that would be of no use. Hence the leaf vertices of $G_{e x}$ are either non-branching or non-extreme branching vertices.

Let $v^{\star}=v^{\star}\left(G_{e x}\right)$ be the unique leaf vertex of $G_{e x}$ reached by traversing down starting from the root and inputting, for all extreme vertices $v$ labeled with $(u, w)$, and going to the right edge if $\nu_{n}\left(P_{u}^{G} \cdot Q_{w}^{G}-P_{w}^{G} \cdot Q_{u}^{G}\right) \in[0, \delta)$ and to the left edge if $\nu_{n}\left(P_{v}^{G} \cdot Q_{v}^{G}-P_{v}^{G} \cdot Q_{v}^{G}\right) \in$ $(1-\delta, 1]$. We call the path from the root to the vertex $v^{\star}$ the dominating path because this is the path that is most likely to be taken if $G$ is run on a random input from $\mathbb{Z}_{n}$ as we make the most likely choice at each equality test (recall that $\delta$ is small). Let $S^{\star}$ denote the straight line program corresponding to this path.

Let $M=\left\lceil\frac{L^{3 / 2}}{(\epsilon / 2)^{5 / 2}}\right\rceil$. Consider Algorithm 2 .

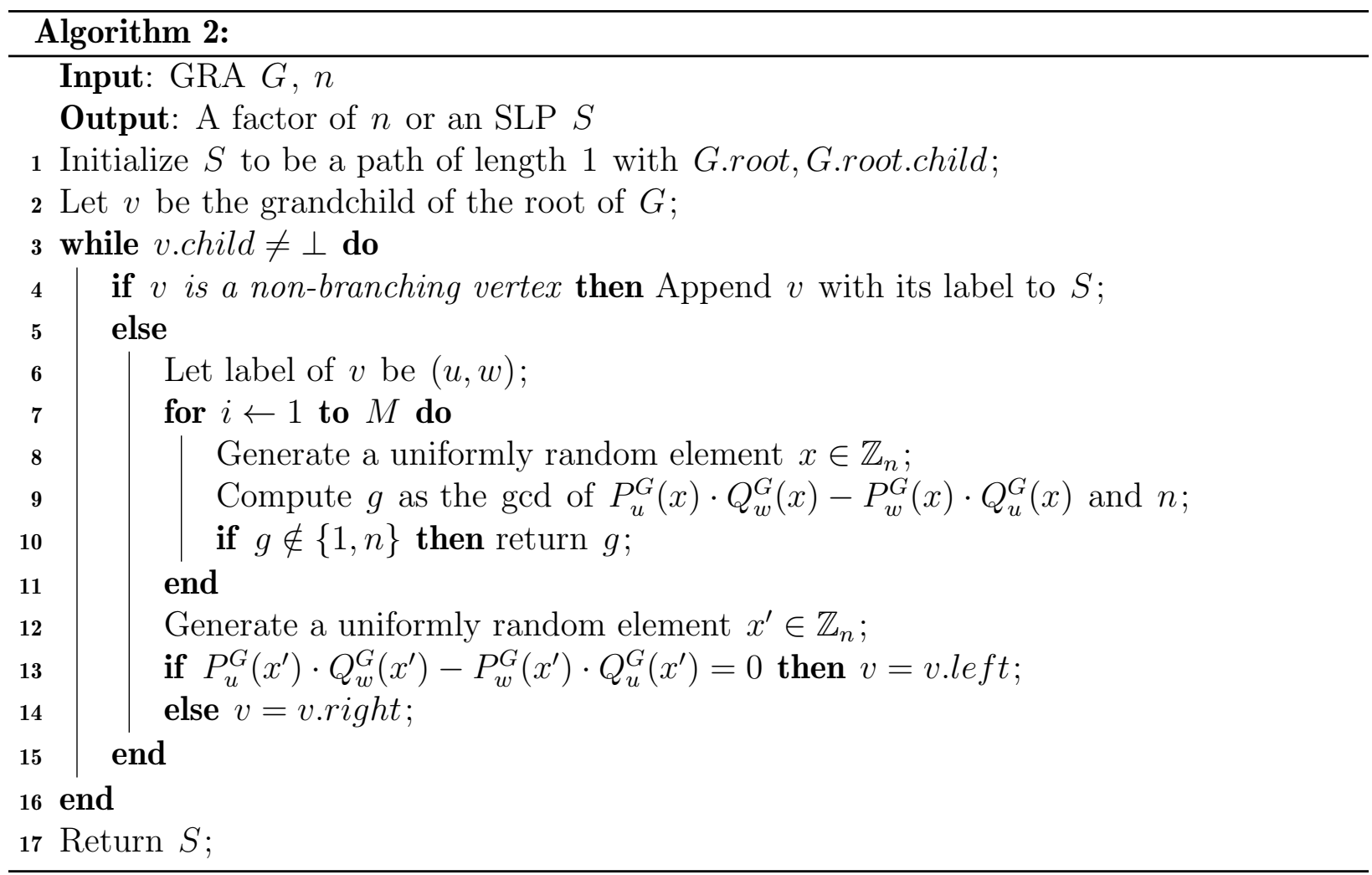

The intuition is that when executing the GRA for a random element $a \in \mathbb{Z}_{n}$, either all the equality test one encounters are irrelevant in the sense that the probability that the 
outcome depends on $a$ is very small, and hence the execution corrresponds to an SLP, or the relevant equality test encountered during the execution can be used to factor.

At each equality query, it tries to find a factor of $n$ using the two pairs of polynomials that are compared. If it fails, it outputs the SLP $S$ as the path from the root to a leaf of $G$ (excluding branching vertices). This path corresponds to a unique path in $G_{e x}$. This path is chosen by generating, for each equality query, a uniformly random element in $\mathbb{Z}_{n}$ and then testing the equality on this element, and choosing the subsequent vertex based on the result of this equality test. Algorithm 2 is successful with high probability (as shown below) if this path is the dominating path, i.e., if it reaches the vertex $v^{\star}$.

Let the leaf vertex of $G_{e x}$ in which this path $S$ terminates be $v_{S}$. Note that $v_{S}$ might not be a leaf vertex of $G$. to $S$.

If Algorithm 2 outputs $S$, then let $\left(P^{S}, Q^{S}\right)$ denote the pair of polynomials corresponding

Let $\mathrm{E}$ be the event that $v_{S}=v^{\star}$, i.e., that the dominating path is found by Algorithm 2 . The event $\mathrm{E}$ does not occur if there exists an extreme vertex $v$ with label $(u, w)$ in the path from the root of $G_{e x}$ to $v_{S}$ such that Algorithm 2 proceeds to the child corresponding to the unlikely output, i.e., goes to left child and $\nu_{n}\left(P_{w}^{G} \cdot Q_{u}^{G}-P_{u}^{G} \cdot Q_{w}^{G}\right) \in[0, \delta)$ or goes to right child and $\nu_{n}\left(P_{w}^{G} \cdot Q_{u}^{G}-P_{u}^{G} \cdot Q_{w}^{G}\right) \in(1-\delta, 1]$. Note that this can happen with probability at most $\delta$ at each extreme vertex $v$ and there can be at most $L$ such extreme vertices in the path from the root of $G_{e x}$ to $v_{S}$. Therefore,

$$
\operatorname{Pr}(\mathrm{E})=1-\operatorname{Pr}(\overline{\mathrm{E}}) \geq 1-\delta \cdot L=1-\frac{\epsilon}{2}
$$

Now we compute the success probability of the algorithm. There are two possible cases depending on whether $v^{\star}$ is a non-extreme vertex or corresponds to a computation operation.

- CASE 1: $v^{\star}$ is a non-extreme vertex.

In this case we show that the factoring algorithm is successful with probability at least $1-\epsilon$.

Let $E^{\prime}$ be the event that Algorithm 2 returns a factor of $n$. We compute $\operatorname{Pr}\left(E^{\prime} \mid E\right)$. If $\mathrm{E}$ holds, then $v_{S}$ is a non-extreme vertex. Therefore, by Lemma 2, a factor of $n$ is returned in one test in Step 10 at the equality query corresponding to $v_{S}$ with probability at least $\delta^{3 / 2}$. The total number of times step 10 is repeated for this equality query is $M$. Therefore ${ }^{7}$,

$$
\operatorname{Pr}\left(\mathrm{E}^{\prime} \mid \mathrm{E}\right) \geq 1-\left(1-\delta^{3 / 2}\right)^{M} \geq 1-\exp \left(-\delta^{3 / 2} M\right)=1-\exp \left(-\frac{2}{\epsilon}\right) \geq 1-\frac{\epsilon}{2} .
$$

This implies

$$
\operatorname{Pr}\left(\mathrm{E}^{\prime}\right) \geq \operatorname{Pr}\left(\mathrm{E}^{\prime} \mid \mathrm{E}\right) \cdot \operatorname{Pr}(\mathrm{E}) \geq\left(1-\frac{\epsilon}{2}\right)^{2} \geq 1-\epsilon .
$$

\footnotetext{
${ }^{7}$ We use the notation $\exp (\cdot)$ to denote exponentiation to the natural base in order to avoid confusion with the public exponent $e$.
} 
- CASE 2: $v^{\star}$ corresponds to a computation operation.

In this case, we show that if the factoring algorithm is not successful, then, with probability $1-\frac{\epsilon}{2}$, we have $\lambda_{n}(S, g) \geq \lambda_{n}(G, g)-\frac{\epsilon}{2} \cdot{ }^{8}$

The fraction of inputs $a \in \mathbb{Z}_{n}$ such that when $G$ is run on $a$, the corresponding path taken on $G_{e x}$ does not terminate in $v^{\star}$ is at most $\delta \cdot L=\frac{\epsilon}{2}$ (because the number of extreme vertices in any path from root to a leaf is at most $L$ ). This implies,

$$
\lambda_{n}\left(S^{\star}, g\right) \geq \lambda_{n}(G, g)-\frac{\epsilon}{2} .
$$

So, if E occurs, then the following event occurs.

$$
\lambda_{n}(S, g) \geq \lambda_{n}(G, g)-\frac{\epsilon}{2} .
$$

Hence,

$$
\operatorname{Pr}\left(\lambda_{n}(S, g) \geq \lambda_{n}(G, g)-\frac{\epsilon}{2}\right) \geq \operatorname{Pr}(\mathrm{E}) \geq 1-\frac{\epsilon}{2} .
$$

The running time of the algorithm is dominated by step 9 which involves evaluating $P_{u}^{G}(x) \cdot Q_{w}^{G}(x)-P_{w}^{G}(x) \cdot Q_{u}^{G}(x)$ for a random input $x$ and then computing its gcd with $n$. This step takes time $O\left(L \log ^{2} n\right)$ and is executed at most $O(L M)$ times. Therefore the time complexity of the algorithm is $O\left(L^{2} \cdot M \cdot \log ^{2} n\right)=O\left(\frac{L^{7 / 2} \log ^{2} n}{\epsilon^{5 / 2}}\right)$.

\subsection{Proof of Theorem 2}

Now, let $g: \mathbb{Z}_{n} \mapsto \mathbb{Z}_{n}$ be the function $a \mapsto a^{1 / e}$. We combine Lemma 4 and Lemma 5 to get the following result.

Corollary 2 For all $\epsilon \in(0,1 / 2]$, there exists an algorithm that takes as input $n=p q$, where $p, q>3$ are primes, an integer $e>1, L \in \mathbb{N}$, and an L-step $G R A G$, runs in time $O\left(\frac{L^{7 / 2} \log ^{2} n}{\epsilon^{5 / 2}}+\log ^{3} e \log ^{2} n\right)$ and returns a factor of $n$ with probability at least $\frac{\lambda_{n}\left(G, x^{1 / e}\right)-\epsilon / 2}{64(L+\log e)}$.

Proof. We first execute Algorithm 2 with input $n, e, G$. If we obtain a factor of $n$, we return this factor. Otherwise, we execute Algorithm 1 on the SLP output by Algorithm 2.

With probability $1-\epsilon$, Algorithm 2 either returns a factor of $n$ or an $L$-step SLP that succeeds with probability $\lambda_{n}\left(G, x^{1 / e}\right)-\epsilon / 2$. In the latter case, Algorithm 1 returns a factor of $n$ with probability $\frac{\lambda_{n}\left(G, x^{1 / e}\right)-\epsilon / 2}{32(L+\log e)}$. Thus, the success probability of the factoring algorithm is at least

$$
(1-\epsilon) \cdot \frac{\lambda_{n}\left(G, x^{1 / e}\right)-\epsilon / 2}{32(L+\log e)} \geq \frac{\lambda_{n}\left(G, x^{1 / e}\right)-\epsilon / 2}{64(L+\log e)} .
$$

\footnotetext{
${ }^{8}$ Note that the straight line program $S$ is a random variable depending on the random choices made by the algorithm.
} 
Now we can conclude the proof of Theorem 2.

Proof. Consider Corollary 2. Let $\epsilon=\frac{\mu}{2} \in(0,1 / 2]$. By linearity of expectation, we have that the probability that the algorithm given in Corollary 2 with input $\mathcal{G}, n, e$ factors $n$ is at least

$$
\frac{\mu}{64(L+\log e)}-\frac{\epsilon / 2}{64(L+\log e)} \geq \frac{\mu}{100(L+\log e)} .
$$

By repeating the algorithm $O((L+\log e) / \mu)$ times, the success probability can be increased to $1 / 2$. The running time of the algorithm is clearly polynomial in $L, \log n, \log e$, and $\frac{1}{\mu}$.

\section{References}

[1] D. Aggarwal and U. Maurer. Breaking RSA generically is equivalent to factoring. In EUROCRYPT 2009, volume 5479 of Lecture Notes in Computer Science, pages 36-53.

[2] K. Altmann, T. Jager and A. Rupp. On Black-Box Ring Extraction and Integer Factorization. In ICALP (2), 2008, volume 5126 of Lecture Notes in Computer Science, pages 437-448.

[3] D. Boneh and R. Lipton. Black box fields and their application to cryptography. In CRYPTO 1996, volume 1109 of Lecture Notes in Computer Science, pages 283-297.

[4] D. Boneh and R. Venkatesan. Breaking RSA may be easier than factoring. In EUROCRYPT 1998, volume 1403 of Lecture Notes in Computer Science, pages 59-71.

[5] D. R. L. Brown. Breaking RSA may be as difficult as factoring. In Cryptology ePrint Archive, Report 205/380, 2006.

[6] I. Damgård and M. Koprowski. Generic lower bounds for root extraction and signature schemes in general groups. In EUROCRYPT 2002, volume 2332 of Lecture Notes in Computer Science, pages 256-271.

[7] W. Diffie and M. Hellman. New directions in cryptography. In IEEE Transactions on Information Theory, volume 22, no. 6, pages 644-654, 1976.

[8] T. Jager. Generic group algorithms. Master's thesis, Ruhr Universität Bochum, 2007.

[9] T. Jager and J. Schwenk. On the analysis of cryptographic assumptions in the generic ring model. In ASIACRYPT 2009, volume 5912 of Lecture Notes in Computer Science, pages 399-416.

[10] A. Joux, D. Naccache and E. Thom. When e-th roots become easier than factoring. In ASIACRYPT 200\%, volume 4833 of Lecture Notes in Computer Science, pages 13-28. 
[11] G. Leander and A. Rupp. On the equivalence of RSA and factoring regarding generic ring algorithms. In ASIACRYPT 2006, volume 4284 of Lecture Notes in Computer Science, pages 241-251.

[12] R. Lidl and H. Niederreiter. In Introduction to finite fields and their applications. Cambridge University Press, 1994.

[13] U. Maurer. Towards proving the equivalence of breaking the Diffie-Hellman protocol and computing discrete logarithms. In CRYPTO 1994, volume 839 of Lecture Notes in Computer Science, pages 271-281.

[14] U. Maurer. Abstract models of computation in cryptoraphy. In Cryptography and Coding 2005, volume 3796 of Lecture Notes in Computer Science, pages 1-12.

[15] U. Maurer and D. Raub. Black-Box Extension Fields and the Inexistence of FieldHomomorphic One-Way Permutations. In ASIACRYPT 200\%, volume 4833 of Lecture Notes in Computer Science, pages 427-443.

[16] U. Maurer and S. Wolf. The relationship between breaking the Diffie-Hellman protocol and computing discrete logarithms. In SIAM Journal of Computing, vol. 28, no. 5, pages 1689-1721, 1999.

[17] V. I. Nechaev. Complexity of a deterministic algorithm for the discrete logarithm. In Mathematical Notes, volume 55, no. 2, pages 91-101, 1994.

[18] R. Rivest, A. Shamir, and L. Adleman. A method for obtaining digital signatures and public key cryptosystems. In Communications of the ACM, volume 21, pages 120-126, 1978.

[19] V. Shoup. Lower bounds for discrete logarithms and related problems. In EUROCRYPT 1997 volume 1233 of Lecture Notes in Computer Science, pages 256-266. 